background. 16/23 (70\%) worked at one of the four previous services. Staff experience of the tender/integration process in terms of 'stress'/'excitement' levels are reported in the Table 1.

\begin{tabular}{|c|c|c|c|c|}
\hline & \multicolumn{4}{|l|}{ Pre } \\
\hline & $\begin{array}{l}09 / 2013 \\
(\%)\end{array}$ & $\begin{array}{l}\text { Months } \\
0-6(\%)\end{array}$ & $\begin{array}{l}\text { Months } \\
7-12(\%)\end{array}$ & $\begin{array}{l}\text { Month } 12 \\
\text { onwards (\% }\end{array}$ \\
\hline Moderate-Very Exciting & $0(0)$ & $1(5)$ & $3(15)$ & $11(50)$ \\
\hline Mildly exciting & $0(0)$ & $0(0)$ & $4(20)$ & $2(9)$ \\
\hline No different & $4(27)$ & $0(0)$ & $2(10)$ & $0(0)$ \\
\hline Mildly stressful & $3(20)$ & $2(10)$ & $3(15)$ & $4(18)$ \\
\hline Moderate-Very Stressful & $9(60)$ & $17(85)$ & $13(65)$ & $7(32)$ \\
\hline Total respondents* & 15 & 20 & 20 & 22 \\
\hline
\end{tabular}

$14 / 22(64 \%)$ of staff believe that SH services should be integrated. 17/22 (77\%) feel patients are now getting a better service (with further improvements needed).

Themes Main 'positives experienced': new skills gained, increasing integration/offer of a 'one-stop-shop' service. Main 'challenges experienced': resistance to change, clash of specialty 'cultures'. The predominant 'suggestion for improvement' was better communication with all staff throughout the process.

Discussion/conclusion The experience of the tender process and early months in the new ISH service was stressful for many staff. This improved with time and staff reported feeling increasingly excited about the new service. Better communication from commissioners and service providers to all staff involved may improve the overall experience of those going through the process in the future.

\section{P185 USING THE STIF PORTFOLIO IN AN "INTEGRATION" TRAINING STRATEGY}

Lee Portas, Ruth Taylor, Ashini Fox*. Nottingham University Hospitals Trust, Nottingham, UK

\subsection{6/sextrans-2015-052126.228}

Background Many UK sexual health clinics are in the process of integrating Sexual and Reproductive Health (SRH) and GUMedicine (GUM) services. Amongst the many challenges they face is that of appropriately training newly integrated staff. Our unit is has recently undergone integration of contraception, termination, outreach and GUM/HIV services. Central to this process was the establishment of a comprehensive training strategy for all clinical staff.

Objectives To describe the successful implementation of an integration training strategy using BASHH's STIF portfolio between 2012-2014.

Methods An initial baseline staff survey demonstrated a lack of consistency of formal sexual health qualifications amongst both SRH and GUM staff. It also highlighted considerable skills amongst some HCAs who had lacked opportunity to formalise them. Our desire was to use existing national qualifications and provide equality of access to all grades of staff.

Results Between 2012-2014 we ran 2 STIF theory courses and 4 STIFLevel 1 assessments. In total 53 staff attended STIF theory and 45 successfully completed STIFLevel 1 (including 8 HCAs). A further 7 senior nurses and $2 \mathrm{SRH}$ doctors have completed STIFIntermediate. One band 7 GUM nurse has also completed STIFAdvanced.

Conclusion The STIF portfolio has provided practical and effective tools in training and assessing staff during our local integration process. We believe that the existence of a clear training strategy helped maintain moral and staff retention during a potentially difficult time and the high level of national qualification amongst our staff will hopefully stand us in good stead in the current commissioning climate.

\section{P186.1 DOES CHLAMYDIA TESTING IN GENERAL PRACTICE MEAN MISSED OPPORTUNITIES FOR THE DIAGNOSIS OF OTHER STIS?: A COMPARISON OF THE POPULATION TESTED IN GENERAL PRACTICE VERSUS SEXUAL HEALTH CLINICS IN BRITAIN}

${ }^{1}$ Soazig Clifton*, ${ }^{1}$ Catherine Mercer, ${ }^{2}$ Jackie Cassell, ${ }^{1}$ Pam Sonnenberg, ${ }^{3}$ Michelle Lu, ${ }^{1,4}$ Sarah Woodhall, ${ }^{4}$ Kate Soldan. ${ }^{1}$ University College London, London, UK; ${ }^{2}$ Brighton and Sussex Medical School, Brighton, UK; ${ }^{3}$ University of Manchester, Manchester, UK; ${ }^{4}$ Public Health England, London, UK

\subsection{6/sextrans-2015-052126.229}

Background Chlamydia testing in general practice may provide opportunities to diagnose those who do not attend sexual health (GUM) clinics. However, as comprehensive STI testing is less likely to be offered in general practice, opportunities could be missed to test, diagnose and treat other STIs including HIV if people at higher sexual risk test in general practice.

Aim To compare demographic, behavioural, and HIV testing characteristics of those tested for chlamydia in general practice with those tested in GUM.

Methods A probability sample survey of the British population undertaken 2010-2012. We analysed weighted data on individuals aged 16-44, reporting at least one sexual partner ever, who reported a chlamydia test in the past year $(\mathrm{n}=1583)$.

Results 26.0\% (24.7-27.4) of women and 16.1\% (14.9-17.3) of men reported testing for chlamydia in the past year, of whom $41.4 \%(38.6-44.2)$ of women and 20.5\% (17.4-24.0) of men tested in general practice. Women tested in general practice were more likely to be older, in a relationship, and to live in rural areas. Men and women tested in general practice reported lower STI risk in terms of (past 5 years): partner numbers, same-sex partners, and overlapping partnerships. Those tested in general practice were less likely to report an HIV test (past 5 years).

Discussion/conclusion While those tested for chlamydia in general practice generally reported lower risk behaviours, rural populations were over-represented, and HIV testing was lower. Pathways to comprehensive STI care need to be universally available for higher risk individuals.

\section{P186 HIGH LEVELS OF USE OF RECREATIONAL DRUGS AND ALCOHOL WITHIN AN INNER LONDON SEXUAL HEALTH CLINIC}

Rosemarie Turner*, Neil Turner, Ann Sullivan, Sara Day. Chelsea and Westminster Hospital, London, UK

\subsection{6/sextrans-2015-052126.230}

Background Drug and alcohol use by patients attending sexual health clinics is not widely assessed as routine. BASHH history taking guidelines and position statement on recreational drug use 